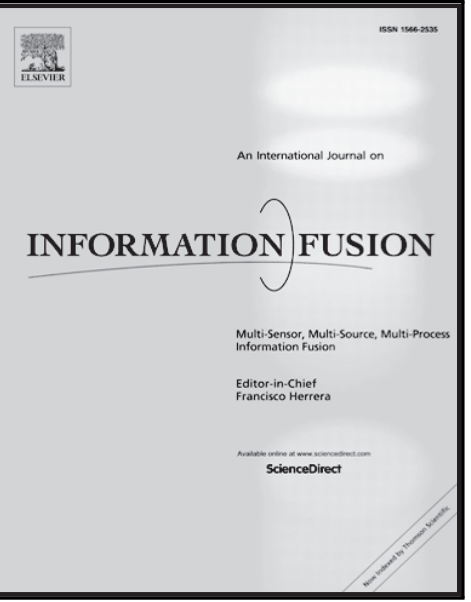

Combining Univariate Approaches for Ensemble Change Detection in Multivariate Data

William J. Faithfull, Juan J. Rodríguez, Ludmila I. Kuncheva

PII: S1566-2535(17)30123-9

DOI: 10.1016/j.inffus.2018.02.003

Reference: INFFUS 958

To appear in: Information Fusion

Received date: 28 February 2017

Revised date: $\quad 17$ January 2018

Accepted date: $\quad 10$ February 2018

Please cite this article as: William J. Faithfull, Juan J. Rodríguez, Ludmila I. Kuncheva, Combining Univariate Approaches for Ensemble Change Detection in Multivariate Data, Information Fusion (2018), doi: 10.1016/j.inffus.2018.02.003

This is a PDF file of an unedited manuscript that has been accepted for publication. As a service to our customers we are providing this early version of the manuscript. The manuscript will undergo copyediting, typesetting, and review of the resulting proof before it is published in its final form. Please note that during the production process errors may be discovered which could affect the content, and all legal disclaimers that apply to the journal pertain. 


\section{Highlights}

- We create and evaluate feature-wise ensembles of univariate change detectors.

- We test 84 ensembles and 3 multivariate detectors on 96 datasets and a case study.

- Ensembles of univariate detectors outperformed established multivariate detectors.

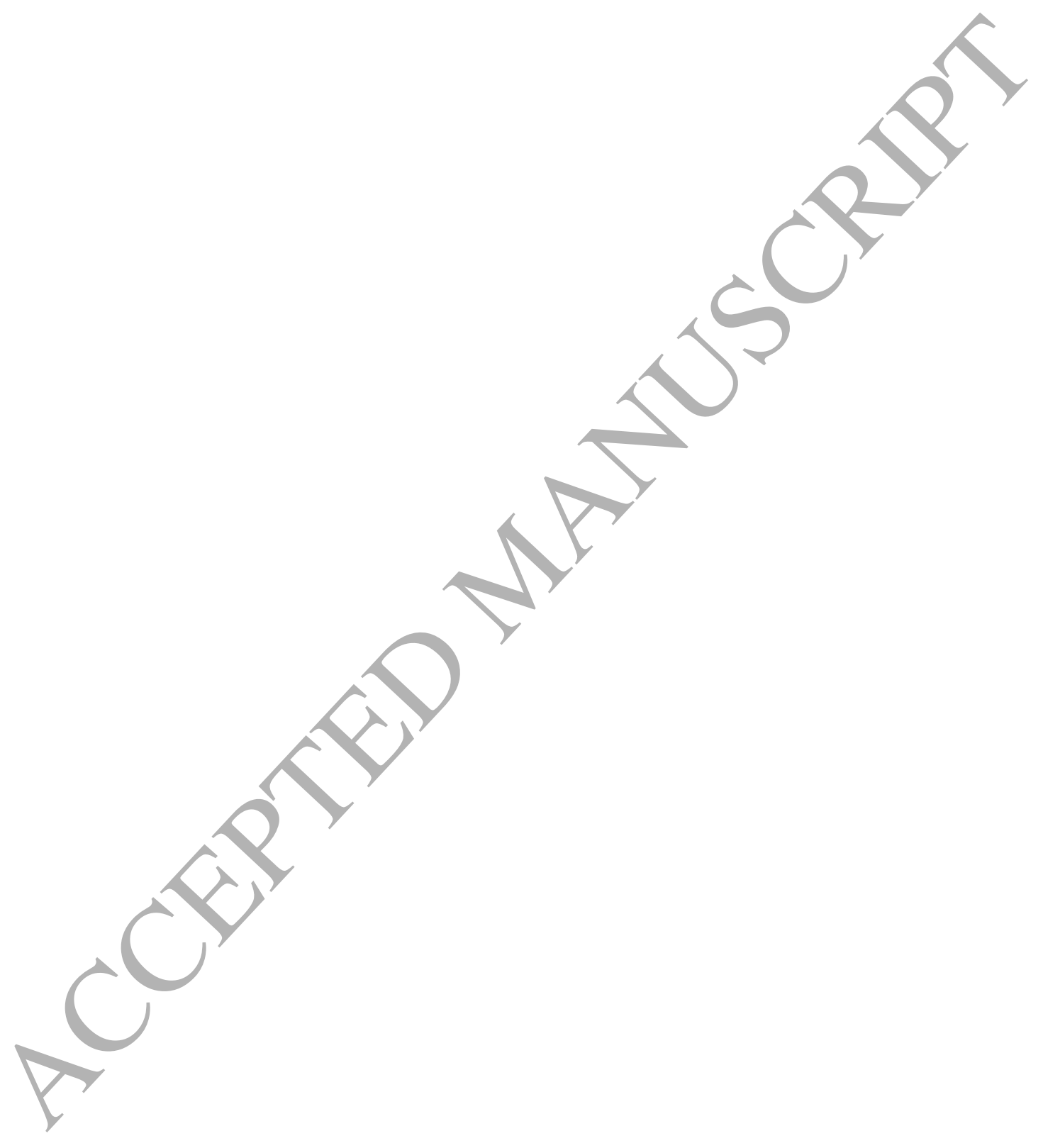




\title{
Combining Univariate Approaches for Ensemble Change Detection in Multivariate Data
}

\author{
William J. Faithfulla,*, Juan J. Rodríguez ${ }^{\mathrm{b}}$, Ludmila I. Kuncheva ${ }^{\mathrm{a}}$ \\ ${ }^{a}$ School of Computer Science, Bangor University, Dean Street, Bangor, Gwynedd, Wales LL57 1UT, UK \\ ${ }^{b}$ Escuela Politécnica Superior, University of Burgos, Avda. de Cantabria s/n, 09006 Burgos, Spain
}

\begin{abstract}
Detecting change in multivariate data is a challenging problem, especially when class labels are not available. There is a large body of research on univariate change detection, notably in control charts developed originally for engineering applications. We evaluate univariate change detection approaches —including those in the MOA framework — built into ensembles where each member observes a feature in the input space of an unsupervised change detection problem. We present a comparison between the ensemble combinations and three established 'pure' multivariate approaches over 96 data sets, and a case study on the KDD Cup 1999 network intrusion detection dataset. We found that ensemble combination of univariate methods consistently outperformed multivariate methods on the four experimental metrics.
\end{abstract}

\section{Introduction}

Change detection is, at its simplest, the task of identifying data points that differ from those seen before. It is often deployed in a supervised or unsupervised context: monitoring the error rate of a learning algorithm which processes the target data, or directly monitoring the target data. In the second context, we do not have class labels with which to estimate an error rate. Unsupervised change detection in a single variable is the univariate case of the problem and has been extensively studied over more than half a century, yielding widely used approaches such as control charts, and specifically, the cumulative sum chart (CUSUM) [1, 2]. There are a variety of univariate methods across the literature from several fields. Basseville and Nikiforov [3] published a monograph on detectors of abrupt change in 1993. There are extensive method reviews in the overlapping field of novelty detection, by Markou and Singh [4] and Pimentel et al. [5], and in outlier detection by Ben-Gal [6]. There are many approaches from the classification literature intended to monitor the error-rate of the incoming data and adapt a deployed classifier accordingly. The MOA (Massive Online Analysis) framework [7, 8] is a popular open source tool for data stream mining, providing a number of approaches for univariate change detection, all of which we evaluate in this work.

We take inspiration from our previous study [9] where we use classifier ensembles to detect concept change in unlabelled multivariate data. We propose an ensemble of univariate detectors (which could be called a 'subspace ensemble') as a means of adapting established univariate change detection methods to multivariate problems. Our hypothesis is that such an ensemble should be competitive or better than 'pure' unsupervised multivariate approaches. We contribute the following: 1 . An evaluation of which established

\footnotetext{
${ }^{*}$ Corresponding Author

Email addresses:

w.faithfull@bangor.ac.uk (William J. Faithfull), jjrodriguez@ubu.es (Juan J. Rodríguez), 1.i.kuncheva@bangor.ac.uk (Ludmila I. Kuncheva)
}

univariate change detection methods are well suited to subspace ensemble combination over 96 common datasets. 2. Whether subspace ensembles outperform three established multivariate change detection methods, especially in high dimensions. 3. A reproducible reinterpretation of the widely used KDD Cup 1999 [10] network intrusion detection dataset as a change detection problem.

When generalising unsupervised change detection to multiple dimensions, the challenges proliferate - in how many features should we expect to see change before signalling? Can we reasonably assume that all features and examples are independent? Multivariate approaches often assume that each example is drawn from a multivariate process $[11,12,13,14]$. Thus, we need not assume that the features are independent. Multivariate change detection attempts to model a multivariate process by means of a function to evaluate the fit of new data (an example or a batch) to that model. Some works monitor components independently (Tartatovsky et al. [15] and Evangelista et al. [16]), meaning that the approach is unable to respond to changes in the correlation of the components. Whether or not this is a disadvantage, depends upon the context of the change.

Change may have a different definition for different problems. For example, if we wish to be alerted when the value of a stock is falling, a sudden rise might be irrelevant. If using a control chart with upper and lower limits, only monitoring the lower limit might considerably lower the false alarm rate. If the problem is well known then a heuristic can be applied, but if that is the case, there is most likely training data available for a supervised approach. Unsupervised approaches must be robust in the face of unknown context. The change we wish to detect could be abrupt or gradual. It could be a single change or repeating concepts. When we move into multiple dimensions, there is even more scope for contextual properties to stretch our assumptions. Change could manifest itself in a single feature, all features, or any number of features in-between. From the novelty detection literature, Evangelista et al. [16] conclude that unsupervised learning in subspaces of the data will typically outperform unsupervised learning that considers the data as a whole. In the course of 
this work, we investigate whether this assertion is reproducible.

The dimensionality of the input data presents a potential challenge. Allipi et. al [17] analyse the effect of an increasing data dimension $d$ on change detectability for log-likelihood based multivariate change detection methods. They demonstrate that in the case of Gaussian random variables, change detectability is upper-bounded by a function that decays as $\frac{1}{d}$. Importantly, the loss in detectability arises from a linear relationship between the variance of the log-likelihood ratio and the data dimension. Evangelista et al. [16] propose that subspace ensembles are also a means to address the curse of dimensionality.

Multivariate detectors treat features as components of an underlying multivariate distribution [11]. We will term such detectors 'pure' multivariate detectors. For pure detectors to work well, the data dimensionality $d$ should not be high, as Allipi et al. argued, and the data coming from the same concept should be available in an i.i.d sequence. This is rarely the case in practice. For example, Tartatovsky et al. [15] observe that the assumption that all examples are i.i.d is very restrictive in the domain of network intrusion detection.

The remainder of the paper is organised as follows. Section 2 covers the background and related work for this problem. Section 3 details the methods used, explains our combination mechanism, and overviews the experimental protocol. Our results are presented in Section 4, and our conclusions follow in Section 5.

\section{Background \& Related Work}

Learning methods are frequently deployed in non-stationary environments, where the concepts may change unpredictably over time. Where class labels are immediately or eventually available, change detection methods can be required to monitor only a univariate error stream from a learner. When a change is detected in the error stream, we can retrain or adapt the model as required. However, when labels are not available, then we cannot use the error rate as a performance indicator. In this instance, a fully unsupervised approach must be taken.

Surveys by Gama et al. [18] and Ditzler et al. [19] discuss the distinction between real and virtual concept drift. Real concept drift is a change in the class conditional probabilities, i.e. the optimal decision boundary. Virtual concept drift refers to a change in the prior probabilities, or distribution of the data. Since in an unsupervised setting, we have no class labels to identify real concept drift, this work would conform to the latter definition. This particular problem formulation is closely related to the assumptions of statistical process control, novelty detection, and outlier detection, for which applications are usually unsupervised, and methods are expected to be applied directly to the domain data.

Most methods for multivariate change detection require two components: a means to estimate the distribution of the incoming data, and a test to evaluate whether new data points fit that model. Estimation of the streaming data distribution is commonly done by either clustering, or multivariate distribution modelling. Gaussian Mixture Models (GMM) are a popular parametric means to model a multivariate process for novelty detection, as in Zorriassatine et al. [12]. Tarassenko et al. [20] and Song et al. [21] use nonparametric Parzen windows (kernel density estimation) to approximate a model against which new data is compared. Dasu et al. [22] construct $k d q$ trees to a similar effect. Krempl et al [23] track the trajectories of online clustering, while Gaber and Yu [24] use the deviation in the clustering results to identify evolution of the data stream. Kuncheva [11] applies $k$ means clustering to the input data and uses the cluster populations to approximate the distribution of the data.

Multivariate statistical tests for comparing distributions such, as Hotelling's t-squared test [25] need to be adapted into the sequential form over time windows of the data [11]. Bespoke statistics continue to be developed for this purpose [13, 14]. Kuncheva [11] introduces a family of log-likelihood ratio detectors which use two time-windows of multivariate data to compute the probability that both are drawn from the same distribution. The observation that log-likelihood based detectors effectively reduce the input space to a univariate statistic can be further exploited, by monitoring that ratio with existing univariate methods [26].

Ensemble methods for monitoring evolving data streams is a growing area of interest within the change detection literature. There are recent surveys on the/subject by Krawczyk et al. [27] and Gomes et al. [28]. The former observe that there has been relatively little research on the combination of drift detection methods. The publications that they review in this area $[29,30]$ deal with the combination of detectors over univariate input data, in contrast to our own formulation. The latter work introduces a taxonomy for data stream ensemble learning methods, and demonstrates the diversity of available methods for ensemble combination. Du et al. [31] utilise an ensemble of change detectors in a supervised approach for a univariate error stream. Alippi et al. [32] introduce hierarchical change detection tests (HCDTs) combining a fast, sequential change detector with a slower, optionally-invoked offline change detector.

In the classification literature, ensemble change detection commonly refers to using these techniques to monitor the accuracy of classifiers in an ensemble, in order to decide when to retrain or replace a classifier [33, 34, 35, 36]. Many of these established univariate methods for change detection are geared towards the supervised scenario which offers a discrete error stream [37, 38]. The Streaming Ensemble Algorithm (SEA) [39] was one of the first of many ensemble approaches for streaming supervised learning problems. However, instead of relying on a change detection, SEA creates an adaptive classifier which is robust to concept drift. Evangelista et al. [16] use a subspace ensemble of one-class Support Vector Machine classifiers in the context of novelty detection. The input space is divided into 3 random subspaces, each monitored by a single ensemble member. Kuncheva [9] uses classifier ensembles to directly detect concept change in unlabeled data, sharing the same problem formulation as this work.

\section{Change detection methods}

The methods we evaluated are detailed in Tables 1 and 2. We chose to evaluate all the univariate detectors offered by MOA [7, 8], an open source project for data stream analysis. Our experiment performs an unsupervised evaluation of all reference implementations of the ChangeDetector interface in the MOA package

$$
\text { moa.classifiers.core.driftdetection }{ }^{1}
$$

\footnotetext{
${ }^{1}$ https://github.com/Waikato/moa/tree/master/moa/src/main/
} 
Table 1: Methods for change detection in univariate data

\begin{tabular}{|c|c|c|}
\hline Method & References & Category \\
\hline SEED & {$[40]$} & Monitoring Distributions \\
\hline ADWIN & {$[41,8]$} & Monitoring Distributions \\
\hline SEQ1 & {$[42]$} & Monitoring Distributions \\
\hline Page-Hinkley & {$[1,8]$} & Sequential Analysis \\
\hline CUSUM1 & [1] & Sequential Analysis \\
\hline CUSUM2 & [8] & Sequential Analysis \\
\hline GEOMA & {$[43,44]$} & Control Chart \\
\hline $\mathrm{HDDM}_{A}$ & [36] & Control Chart \\
\hline EDDM & {$[38,8]$} & Control Chart \\
\hline DDM & {$[37,8]$} & Control Chart \\
\hline EWMA & {$[43,8,44]$} & Control Chart \\
\hline $\mathrm{HDDM}_{W}$ & [36] & Control Chart \\
\hline
\end{tabular}

Table 2: Methods for change detection in multivariate data

\begin{tabular}{rcc} 
Method & References & Category \\
\hline SPLL & {$[11]$} & Monitoring Distributions \\
Log-likelihood KL & {$[11]$} & Monitoring Distributions \\
Log-likelihood Hotelling & {$[11]$} & Monitoring Distributions \\
\hline
\end{tabular}

The interface contract implies the following basic methods to provide an input and subsequently check if change was detected:

$$
\begin{aligned}
& \text { public void input(double inputValue); } \\
& \text { public boolean getChange(); }
\end{aligned}
$$

All the univariate detectors are provided by MOA except CUSUM1, which is a CUSUM chart with upper and lower limits which was implemented in Java, and integrated into the experiment to serve as a baseline. We arrive at a final figure of 88 detectors, 3 of which are the multivariate approaches listed in Table 2, and the remaining 85 are ensembles of the univariate approaches with varying thresholds. The experimental details will be given in subsection 3.2. A full list of the 96 datasets and their characteristics can be found in Table 4. Our metrics for evaluation and our experimental protocol are addressed in subsection 3.3. Finally, we discuss the case study in subsection 3.4.

\subsection{Overview of the methods}

The univariate detectors are listed in Table 1, with their accompanying publications. We categorise the methods based on the change detection taxonomy presented in Gama et. al [18]. What follows is a high-level overyiew of the theory behind each category of methods along with an abridged description of each detector. More details for each detector can be found in the accompanying publications in Table 1. The source code for each detector is available for inspection in the MOA repository.

\subsubsection{Sequential Analysis}

Sequential analysis methods have much in common with the Sequential Probability Ratio Test (SPRT) [2]. Consider a sequence of examples $X=\left[x_{1}, \ldots, x_{N}\right]$. The null hypothesis $H_{0}$ is that $X$ is generated from a given distribution $p_{0}(x)$, and the alternative

$\mathrm{java} / \mathrm{moa} / \mathrm{classifiers/core/driftdetection}$ hypothesis $H_{1}$ is that $X$ is generated from another (known) distribution $p_{1}(x)$. The logarithm of the likelihood ratio for the two distributions is calculated as

$$
\Lambda_{N}=\sum_{i=1}^{N} \log \frac{p_{1}\left(x_{i}\right)}{p_{0}\left(x_{i}\right)}
$$

Two thresholds, $\alpha$ and $\beta$ are defined depending on the target error rates. If $\Lambda_{N}<\alpha, H_{0}$ is accepted, else if $\Lambda_{N}>\beta, H_{1}$ is accepted. In the case where $\alpha<=\Lambda_{N}<=\beta$, the decision is postponed, the next example in the stream, $x_{N+1}$, is added to the set, and $\Lambda_{N+1}$ is calculated and compared with the thresholds. Cumulative sum (CUSUM) [1] is a sequential analysis technique based on the same principle. The test is widely used for detecting significant change in the mean of input data. Starting with an upper cumulative sum statistic $g \triangle_{0}=0$, CUSUM updates $g \triangle$ for each subsequent example as

$$
g \triangle_{i}=\max \left(0, g \triangle_{i-1}+\left(x_{i}-\delta\right)\right)
$$

where $\delta$ is the magnitude of acceptable change. Change is signalled when $g \triangle_{i}>\lambda$, where $\lambda$ is a fixed threshold. If we wish to detect both positive and negative shifts in the mean, we can also compute and threshold the lower sum as

$$
g \nabla_{i}=\min \left(0, g \nabla_{i-1}-\left(x_{i}-\delta\right)\right)
$$

The Page-Hinkley test [1] is derived from CUSUM, and adapted to detect an abrupt change in the average of a Gaussian process $[18,45]$.

\subsubsection{Control Charts}

Control charts ${ }^{2}$ are a category of methods that are based upon Statistical Process Control (SPC). In SPC, the modus operandi is to consider the problem as a known statistical process, and monitor its evolution. Assume that we monitor classification error. This error can be interpreted as a Bernoulli random variable with probability of "success" (where error occurs) $p$. The probability is unknown at the start of the monitoring, and is re-estimated with every new example as the proportion of errors encountered thus far. At example $i$, we have a binomial random variable with estimated probability $p_{i}$ and standard deviation $\sigma_{i}=\sqrt{p_{i}\left(1-p_{i}\right) / i}$. One way to use this estimate is described below $[37,18]$ :

1. Denote the (binary) streaming examples as $x_{1}, x_{2}, \ldots$. To keep a running score of the minimum $p$, start with estimate $p_{\min }=1$, and $\sigma_{\min }=0$. Initialise the stream counter $i \leftarrow 1$.

2. Observe $x_{i}$. Calculate $p_{i}$ and $\sigma_{i}$. For an error and a standard deviation $\left(p_{i}, \sigma_{i}\right)$ at example $x_{i}$, the method follows a set of rules to place itself into one of three possible states: in control, warning, and out of control. Under the commonly used confidence levels of $95 \%$ and $99 \%$, the rules are:

\footnotetext{
${ }^{2}$ A number of the control chart methods in MOA are intended for supervised predictive error monitoring rather than continuous data., however they accept continuous data by virtue of the ChangeDetector interface. While their assumptions are violated by the unsupervised experiment, we include their results for demonstrative purposes as MOA does not make a distinction. The Page-Hinkley detector might be expected to perform better on a prequential error stream [46], but retains valid assumptions for unsupervised features.
} 
- If $p_{i}+\sigma_{i}<p_{\min }+2 \sigma_{\min }$, then the process is deemed to be in control.

- If $p_{i}+\sigma_{i} \geq p_{\min }+3 \sigma_{\min }$, then the process is deemed to be out of control.

- If $p_{\text {min }}+2 \sigma_{\text {min }} \leq p_{i}+\sigma_{i}<p_{\text {min }}+3 \sigma_{\text {min }}$, then this is considered to be the warning state.

3. If $p_{i}+\sigma_{i}<p_{\min }+\sigma_{\min }$, re-assign the minimum values: $p_{\min } \leftarrow p_{i}$ and $\sigma_{\min } \leftarrow \sigma_{i}$.

4. $i \leftarrow i+1$. Continue from 2 .

The geometric moving average chart (GEOMMA), introduced by Roberts [44], assigns weights to each observation such that the weight of older observations decreases in geometric progression. This biases the method towards newer observations, improving the adaptability. Exponentially Weighted Moving Average (EWMA) charts are a progression of this approach such that the rate of weight decay is continuous and can be tuned.

The EWMA charts used by Ross et al. [43] expect the initial distribution to have known parameters, which is a restrictive assumption in the area of change detection. To address this limitation, the initial distribution is approximated in advance through regression of the distributional parameters to achieve a desired Average Running Length (ARL).

Drift Detection Method (DDM) [37] is designed to monitor classification error using a control chart construction. It assumes that the error rate will decrease while the underlying distribution is stationary.

Similarly, the Early Drift Detection Method (EDDM) [38] is an extension of DDM which takes into account the time distance between errors as opposed to considering only the magnitude of the difference, which is aimed at improving the performance of the detector on gradual change. $\mathrm{HDDM}_{A}$ and $\mathrm{HDDM}_{W}$ are extensions which remove assumptions relating the to probability density functions of the error of the learner. Instead, they assume that the input is an independent and bounded random variable, and use Hoeffding's inequality to compute the bounds [36].

\subsubsection{Monitoring two distributions}

The methods in this category monitor the distributions of two windows of data. The basic construction involves a reference window composed of old data, and a detection window composed of new data. This can be achieved with a static reference window and a sliding detection window, or a sliding pair of windows over consecutive observations. The old and new windows can be compared with statistical tests, with the null hypothesis being that both windows are drawn from the same distribution.

For fixed-sized windows, their sizes need to be decided $a$ priori, which poses a problem. A small-sized window discards old examples swiftly, best representing the current state, but it also makes the method vulnerable to outliers. Conversely, a large-sized window provides more stable estimates of the probabilities and other variables of interest, but takes longer to pick up a change. In order to address this selection problem, there are a number of approaches for growing and shrinking sliding windows on the fly [41, 47, 48].

A widely-used approach of this type is Adaptive Windowing (ADWIN) by Bifet and Gavaldà [41]. It keeps a variable-length window of recently seen examples, and a fixed-size reference window.
For the variable size window, ADWIN keeps the longest possible window within which there has been no statistically significant change. In its formulation as a change detector, change is signalled when the difference of the averages of the windows exceeds a computed threshold. When this threshold is reached, the reference window is emptied, and replaced by the variable length window, which is then regrown from subsequent observations. The SEQ1 algorithm [42] is an evolution of the ADWIN approach with a lower computational complexity. Cut-points are computed differently - where ADWIN makes multiple passes through the window to compute candidate cut-points, SEQ1 only examines the boundary between the latest and previous batch of elements. Secondly, the means of data segments are estimated through random sampling instead of exponential histograms. Finally, the authors employ the Bernstein bound instead of the Hoeffding bound to establish whether two sub-windows are drawn from the same population because the Hoeffding bound was deemed to be overly conservative.

In the SEED algorithm by Huang et al. [40], the data comes in blocks of a fixed size, so the candidate change points are the block's starting and ending points. Adjacent blocks are examined and grouped together if they are deemed sufficiently similar. This operation, termed 'block compression', removes candidate change points which have a lower probability of being true change points. Pooling blocks together amounts to obtaining larger windows, which in turn, ensures more stable estimates of the probabilities of interest compared to estimates from the original blocks. Drift detection is subsequently carried out by analysing possible splits between the newly-formed blocks.

\subsubsection{Multivariate change detectors}

Consider a random vector $\mathbf{x}$

$$
\mathbf{x}=\left[x_{1}, x_{2}, \ldots, x_{n}\right]^{T} \in \mathbb{R}^{n},
$$

drawn from a continuous stream

$$
\mathbf{x}_{i}, \mathbf{x}_{i+1}, \ldots, \mathbf{x}_{N} \ldots
$$

We assume that $\mathbf{x}$ are drawn from a probability distribution $p_{0}(\mathbf{x})$ up to a certain point $c$ in the stream, and from a different distribution thereafter. The objective is to find the change point $c$. We can estimate $p_{0}$ from the incoming examples and compute the likelihood $\mathcal{L}\left(\mathbf{x} \mid p_{0}\right)$ for subsequent examples. A successful detection algorithm will be able to identify $c$ by a decrease of the likelihood of the examples arriving after $c$. To estimate and compare the likelihoods before and after a candidate point, the data is partitioned into a pair of adjacent sliding time-windows of examples, $W_{1}$ and $W_{2}$.

The Hotelling detector uses the multivariate $T^{2}$ test for equal means, and assumes equal covariance matrices of $W_{1}$ and $W_{2}$. Therefore, if the change of the distribution comes from change in the variances or covariances in the multidimensional space of the data, the test will be powerless.

As an alternative, we used a non-parametric change detector based on the Kullback-Leibler divergence (KL). To this end, the data in $W_{1}$ is clustered using k-means into $K$ clusters, $C=\left\{C_{1}, \ldots, C_{K}\right\}$. A discrete distribution $P$ is defined on $C$, where each cluster is given a probability equal to the proportion of examples it holds. The examples in $W_{2}$ are labelled in the $K$ clusters by the nearest cluster 


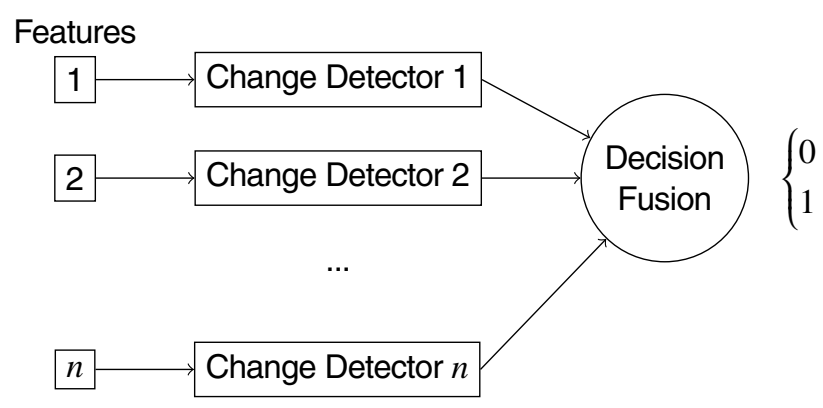

Figure 1: An illustration of the ensemble combination scheme. All change detectors are of the same type, but each monitors a different feature.

centroid. The proportions of examples labelled in the respective cluster define the distribution $Q$ over $C$, this time derived from the data in $W_{2}$. If the two distributions were identical, the $\mathrm{KL}$ divergence will be close to 0 , and if they are very different, it will be close to 1 . The success on this detector depends on a wise choice of the number of clusters $K$ relative to the window sizes and the space dimensionality $n$. A smaller number of clusters ensures that there are enough points in each cluster to allow for reasonable estimates of the probability mass function. On the other hand, a larger number of clusters allows for better fidelity in approximating the distributions.

Finally, we include in the experiment the Semi-Parametric LogLikelihood detector (SPLL) [11] as a compromise between the parametric detector (Hotelling) and non-parametric detector (KL). SPLL, like KL, applies k-means clustering to $W_{1}$ into $K$ clusters. However, rather than approximating a discrete distribution, the criterion function of SPLL is derived assuming that we have fitted a Gaussian mixture with equal mixing proportion and common covariance matrix for the $K$ clusters. The first part of the statistic of the SPLL detector is proportional to the mean of the squared Mahalanobis distances between each example in $W_{2}$ and its nearest cluster centroid. The calculation is repeated symmetrically by clustering first $W_{2}$, and then assigning labels to the examples in $W_{1}$. This gives the second part of the SPLL statistic. These two parts are subsequently averaged. ${ }^{3}$

\subsection{Ensemble combination of univariate detectors}

In order to evaluate univariate approaches on multivariate data, we adopted an ensemble combination strategy whereby each member monitors a single feature of the input space. This approach is analogous to using a subspaces ensemble method with a subspace size of 1 , with as many subspaces and detectors as the dimensionality of the input space. Using subspaces with a size greater than 1, as in Evangelista et al. [16], would require combination of multivariate approaches. Figure 1 shows an illustration of the ensemble combination scheme. In this set of experiments, the decisions are combined by a simple voting scheme with a variable threshold. Our naming convention for a single ensemble is as follows:

\section{DETECTOR - AGREEMENT THRESHOLD}

For example, ADWIN-30 refers to an ensemble of univariate ADWIN detectors, which requires $30 \%$ agreement at any given

\footnotetext{
${ }^{3}$ MATLAB code is available at

https://github.com/LucyKuncheva/Change-detection
}

point to signal change. The multivariate detectors will simply be referred to as, KL, SPLL and Hotelling, as they are not ensembles. ${ }^{4}$

Diversity is an important consideration when building an ensemble, because it implies that the members will make different mistakes $[49,50]$ and there have been several analyses of ensemble diversity in evolving data streams [51, 28]. However, unlike in these works, our ensembles consist of identical detectors. Diversity is introduced through the differing input to each detector. On a related note, there will be redundant features in the datasets, which will effect ensemble performance. Ideally this would be addressed through a feature extraction step, but such a measure is both difficult to generalise across datasets and outside the scope of this paper. As our ensembles are created with identical members, no one type of detector can gain an advantage in the results due to drawing many redundant features by chance.

\subsection{Experimental protocol}

The main experiment of this paper evaluates our multivariate change detection methods across the 96 datasets in Table 4 . We evaluate the 3 multivariate detectors - SPLL, KL and Hotelling, an ensemble of these multivariate detectors, and 84 feature-wise ensembles of the univariate detectors with varying agreement thresholds, making a total of 88 detectors. A breakdown of the methods is presented in Table 3.

We note that when the thresholds in Table 3 are utilised on particularly small ensembles, the lower thresholds will become logically equivalent. For example, in ensembles with fewer than 20 members, the $5 \%$ and $1 \%$ thresholds will make the same decisions $(20 \times 0.5=1)$. Since $43.33 \%$ of the datasets have more than 20 features, the difference in results between these lower thresholds will depend upon the larger datasets.

All the methods were evaluated against three rates of change: Abrupt, Gradual 100 and Gradual 300, for which we recorded separate sets of results. Algorithm 1 is a simplified pseudocode representation of the experiment. For each leg of the experiment, each detector is evaluated 100 times for each dataset. On each of these runs, we choose a random subset of the classes, and take this subset to represent distribution $p_{0}$ (before the change). The subset with the remaining classes is taken to represent distribution $p_{1}$ (after the change). Points are then sampled randomly, with replacement, from the $p_{0}$ and $p_{1}$ sets -500 examples in the abrupt case, 600 and 800 respectively in the gradual cases. Denote these samples by $S_{1}$ and $S_{2}$, respectively. We add a small random value to each example, scaled by the standard deviation of the data, to avoid examples that are exact replicas. In the abrupt case, $S_{1}$ and $S_{2}$ are concatenated to create a 1000 -example test sample with with i.i.d stream from index 1 to 500, coming from $p_{0}$, followed by an abrupt change at index 500 to another i.i.d. stream of examples coming from $p_{1}$. To emulate gradual change over 100 examples, we take $S_{1}$ and $S_{2}$ as before, but do not concatenate them. At index 500, we sample with increasing frequency from $S_{2}$. The chance of an example

\footnotetext{
${ }^{4}$ The ensemble of multivariate detectors is a special case, because, unlike the ensembles of univariate detectors, it consists of only three detectors. In this case, the number of members does not scale with the number of features. As such, there is no benefit in having a scale of agreement thresholds when there are only ever 3 ensemble members. We chose $50 \%$ as a simple majority out of 3 .
} 
Table 3: The ensembles and detectors evaluated in the experiment

\begin{tabular}{|c|c|c|}
\hline Ensemble & Agreement Thresholds & Count \\
\hline SEED & $1,5,10,20,30,40,50$ & 7 \\
\hline ADWIN & $1,5,10,20,30,40,50$ & 7 \\
\hline SEQ1 & $1,5,10,20,30,40,50$ & 7 \\
\hline $\mathrm{PH}$ & $1,5,10,20,30,40,50$ & 7 \\
\hline CUSUM1 & $1,5,10,20,30,40,50$ & 7 \\
\hline CUSUM2 & $1,5,10,20,30,40,50$ & 7 \\
\hline GEOMMA & $1,5,10,20,30,40,50$ & 7 \\
\hline $\mathrm{HDDM}_{A}$ & $1,5,10,20,30,40,50$ & 7 \\
\hline EDDM & $1,5,10,20,30,40,50$ & 7 \\
\hline DDM & $1,5,10,20,30,40,50$ & 7 \\
\hline EWMA & $1,5,10,20,30,40,50$ & 7 \\
\hline $\mathrm{HDDM}_{W}$ & $1,5,10,20,30,40,50$ & 7 \\
\hline \multirow[t]{6}{*}{ MV } & 50 & 1 \\
\hline & & $\begin{array}{c}\text { Total } \\
85\end{array}$ \\
\hline & Multivariate Detector & Count \\
\hline & SPLL & 1 \\
\hline & KL & 1 \\
\hline & Hotelling & 1 \\
\hline & & $\begin{array}{c}\text { Total } \\
3\end{array}$ \\
\hline
\end{tabular}

coming from $S_{1}$ increases linearly from $1 \%$ at index 501 to $100 \%$ at index 600. Note that the class subsets for sampling $S_{1}$ and $S_{2}$ were chosen randomly for each of the 100 runs of the experiment.

As the chosen datasets are not originally intended as streaming data, our experiment uses the concept that the separable characteristics of each class are woven throughout the features. Therefore some changes will be easier to detect than others, introducing variety in our test data. Even if the sample size is insufficient to detect changes in a given dataset, this does not compromise experimental integrity because every detector faces the same challenge. A detector which performs well on average has negotiated a diverse range of class separabilities.

Datasets with fewer than 1000 examples will be oversampled in this experiment, but we found no relationship between the oversampling percentage of a dataset and our results. Even if this were to hinder or benefit the task at hand, the challenge is the same for every detector.

We measure the following characteristics for each method, averaged over the 100 runs each, for abrupt and gradual change on each dataset:

ARL Average Running Length: The average number of contiguous observations for which the detector did not signal change.

TTD Time To Detection: The average number of observations between a change occurring and the detector signalling.

NFA The percentage of runs for which the detector did not issue a false alarm.

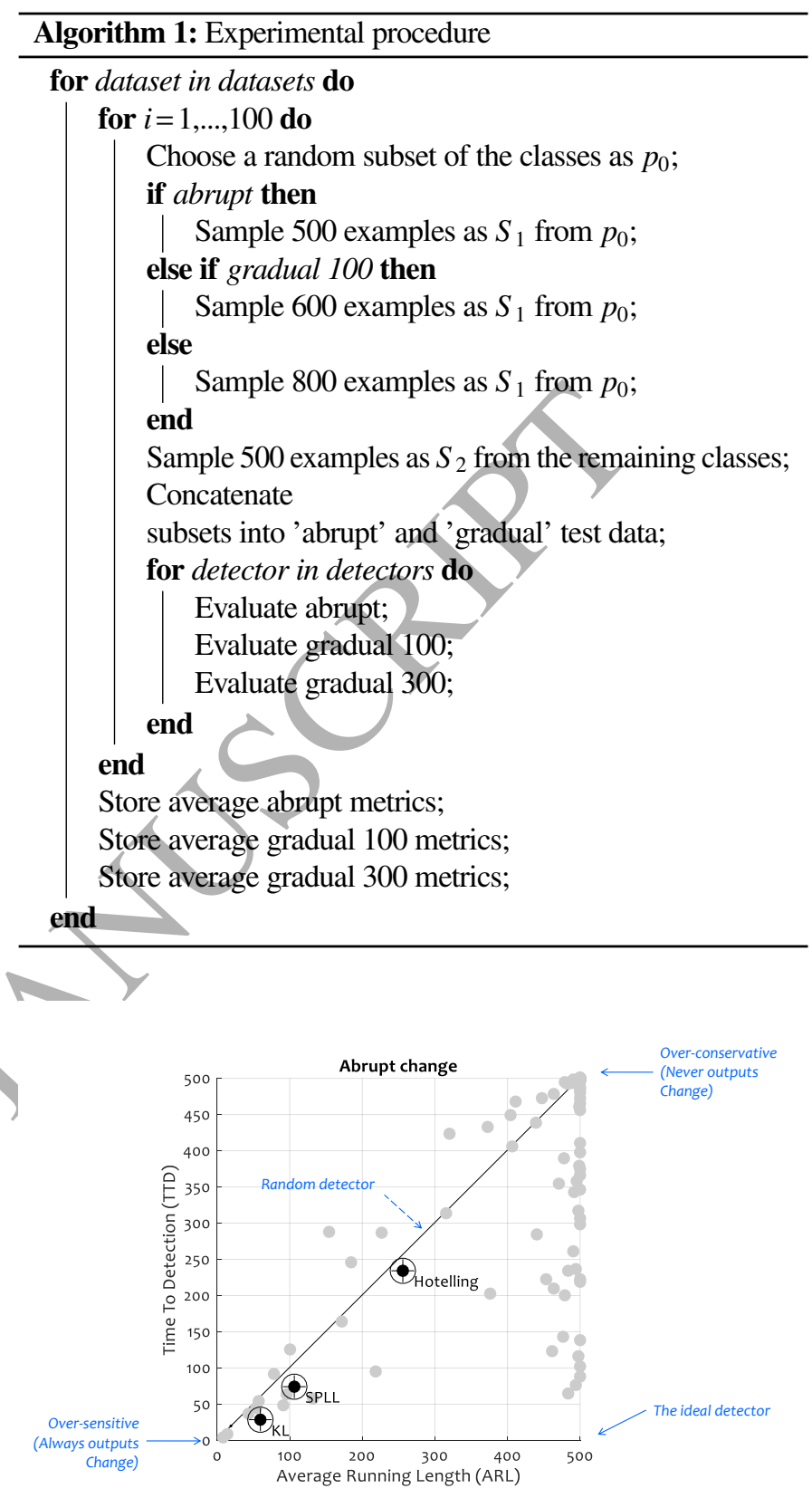

Figure 2: Scatterplot of the 88 detector methods in the space $(A R L, T T D)$ for the Abrupt-change part of the experiment. The three individual detectors are highlighted.

MDR The percentage of runs for which the detector did not signal after a true change.

Based on these characteristics, a good method should maximise $A R L$ and NFA, and minimise TTD and MDR.

Figure 2 is the archetype of our result figures. It plots $T T D$ versus $A R L$ for the detection methods. The grey dots correspond to ensemble methods, and the highlighted black dots correspond to the individual detectors (Hotelling, KL, and SPLL). The ideal detector will have $A R L=\infty$ (500 in our experiment, meaning that no false detection has been made before the true change happened), and $T T D=0$. This detector occupies the bottom right corner of the plot. 
Table 4: The 96 datasets used in the main experiment.

$N$ is examples, $n$ is features and $c$ is classes.

\begin{tabular}{|c|c|c|c|c|c|c|c|}
\hline dataset & $N$ & $n$ & $c$ & dataset & $N$ & $n$ & $c$ \\
\hline abalone & 4177 & 8 & 3 & molec-biol-splice & 3190 & 60 & 3 \\
\hline acute-inflammation & 120 & 6 & 2 & monks-1 & 556 & 6 & 2 \\
\hline acute-nephritis & 120 & 6 & 2 & monks-2 & 601 & 6 & 2 \\
\hline adult & 48842 & 14 & 2 & monks-3 & 554 & 6 & 2 \\
\hline annealing & 850 & 31 & 3 & mushroom & 8124 & 21 & 2 \\
\hline arrhythmia & 295 & 262 & 2 & musk-1 & 476 & 166 & 2 \\
\hline balance-scale & 576 & 4 & 2 & musk-2 & 6598 & 166 & 2 \\
\hline bank & 4521 & 16 & 2 & nursery & 12958 & 8 & 4 \\
\hline blood & 748 & 4 & 2 & oocytes_merluccius_nucleus_4d & 1022 & 41 & 2 \\
\hline breast-cancer & 286 & 9 & 2 & oocytes_merluccius_states_2f & 1022 & 25 & 3 \\
\hline breast-cancer-wisc & 699 & 9 & 2 & oocytes_trisopterus_nucleus_2f & 912 & 25 & 2 \\
\hline breast-cancer-wisc-diag & 569 & 30 & 2 & oocytes_trisopterus_states_5b & 898 & 32 & 2 \\
\hline car & 1728 & 6 & 4 & & 5620 & 62 & 10 \\
\hline cardiotocography-10clases & 2126 & 21 & 10 & ozone & 2536 & 72 & 2 \\
\hline cardiotocography-3clases & 2126 & 21 & 3 & page-blocks & 5445 & 10 & 4 \\
\hline chess-krvk & 28029 & 6 & 17 & pendigits & 10992 & 16 & 10 \\
\hline chess-krvkp & 3196 & 36 & 2 & pima & 768 & 8 & 2 \\
\hline congressional-voting & 435 & 16 & 2 & planning & 182 & 12 & 2 \\
\hline conn-bench-sonar-mines-rocks & 208 & 60 & 2 & ringnorm & 7400 & 20 & 2 \\
\hline conn-bench-vowel-deterding & 990 & 11 & 11 & seeds & 210 & 7 & 3 \\
\hline connect-4 & 67557 & 42 & 2 & semeion & 1593 & 256 & 10 \\
\hline contrac & 1473 & 9 & 3 & soybean & 362 & 35 & 4 \\
\hline credit-approval & 690 & 15 & 2 & spambase & 4601 & 57 & 2 \\
\hline cylinder-bands & 512 & 35 & 2 & spect & 265 & 22 & 2 \\
\hline dermatology & 297 & 34 & 4 & spectf & 267 & 44 & 2 \\
\hline ecoli & 272 & 7 & 3 & statlog-australian-credit & 690 & 14 & 2 \\
\hline energy-y1 & 768 & 8 & 3 & statlog-german-credit & 1000 & 24 & 2 \\
\hline energy-y2 & 768 & 8 & 3 & statlog-heart & 270 & 13 & 2 \\
\hline glass & 146 & 9 & 2 & statlog-image & 2310 & 18 & 7 \\
\hline haberman-survival & 306 & 3 & 2 & statlog-landsat & 6435 & 36 & 6 \\
\hline hayes-roth & 129 & 3 & 2 & statlog-shuttle & 57977 & 9 & 5 \\
\hline heart-cleveland & 219 & 13 & 2 & statlog-vehicle & 846 & 18 & 4 \\
\hline heart-hungarian & 294 & 12 & 2 & steel-plates & 1941 & 27 & 7 \\
\hline heart-va & 107 & 12 & 2 & synthetic-control & 600 & 60 & 6 \\
\hline hill-valley & 1212 & 100 & 2 & teaching & 102 & 5 & 2 \\
\hline horse-colic & 368 & 25 & 2 & thyroid & 7200 & 21 & 3 \\
\hline ilpd-indian-liver & 583 & 9 & 2 & tic-tac-toe & 958 & 9 & 2 \\
\hline imalge-segmentation & 2310 & 18 & 7 & titanic & 2201 & 3 & 2 \\
\hline ionosphere & 351 & 33 & 2 & twonorm & 7400 & 20 & 2 \\
\hline iris & 150 & 4 & 3 & vertebral-column-2clases & 310 & 6 & 2 \\
\hline led-display & 1000 & 7 & 10 & vertebral-column-3clases & 310 & 6 & 3 \\
\hline letter & 20000 & 16 & 26 & wall-following & 5456 & 24 & 4 \\
\hline low-res-spect & 469 & 100 & 3 & waveform & 5000 & 21 & 3 \\
\hline lymphography & 142 & 18 & 2 & waveform-noise & 5000 & 40 & 3 \\
\hline magic & 19020 & 10 & 2 & wine & 130 & 13 & 2 \\
\hline mammographic & 961 & 5 & 2 & wine-quality-red & 1571 & 11 & 4 \\
\hline miniboone & 130064 & 50 & 2 & wine-quality-white & 4873 & 11 & 5 \\
\hline molec-biol-promoter & 106 & 57 & 2 & yeast & 1350 & 8 & 5 \\
\hline
\end{tabular}


Dots which are close to this corner are indicative of good detectors.

The two trivial detectors lie at the two ends of the diagonal plotted in the figure. A detector which always signals change has $A R L=0$ and $T T D=0$, while detector which never signals change has $A R L=$ 500 and $T T D=500$. A detector which signals change at random will have its corresponding point on the same diagonal. The exact position on the diagonal will depend on the probability of signalling a change (unrelated to actual change). Denote this probability by $p$. Then $A R L$ is the expectation of a random variable $X$ with a geometric distribution ( $X$ is the number of Bernoulli trials needed to get one success, with probability of success $p$ ), that is $A R L=\frac{1-p}{p}$. The time to detection, $T T D$, amounts to the same quantity because it is also the expected number of trials to the first success, with the same probability of success $p$. Thus the diagonal $A R L=T T D$ is a baseline for comparing change detectors. A detector whose point lies above the diagonal is inadequate; it detects change when there is none, and fails to detect an existing change. We follow the same archetype for visualisation of the MDR/NFA space. We plot MDR against 1-NFA for these figures in order to maintain the same visual orientation for performance. Therefore the ideal detector in this space is also at point $(1,0)$, i.e., all changes were detected, and there were no false alarms.

\subsection{A Case Study}

In addition to the main experiment, we conducted a practical case study on a network intrusion detection dataset. We chose the popular KDD Cup 1999 intrusion detection dataset, which is available from the UCI Machine Learning Repository [10]. With a network intrusion dataset, the change context is more likely to be longer-lived change from one concept to another, which could be either abrupt or gradual. The dataset consists of 4,900,000 examples and 42 features extracted from seven-weeks of TCP dump data from network traffic on a U.S. Air Force LAN. During the seven weeks, the network was deliberately peppered with attacks which fall into four main categories.

- Denial of Service (DOS): An attacker overwhelms computing resources in order to deny access to them.

- Remote to Login (R2L): Attempts at unauthorised access from a remote machine, such as guessing passwords.

- Unauthorized to Root (U2R): Unauthorised access to local superuser privileges, through a buffer overflow attack, for example.

- Probing: Surveillance and investigation of weaknesses, such as port scanning.

Of these categories, there are 24 specific attack concepts, or 24 classes. This dataset is most commonly interpreted as a classification task. Viewed as such, it offers some interesting challenges in its deficiencies. For example, there is $75 \%$ and $78 \%$ redundancy in duplicated records across the training and testing set respectively [52]. This can serve to bias learning algorithms toward frequent records. It also has very imbalanced classes, with the smurf and neptune DoS attacks constituting $71 \%$ of the data points; more than the 'normal' class. We offer an interpretation of this data as a change detection task.

We evaluated the methods on the testing dataset. Since the data is sequential, we pass observations in order, one-by-one to each of the detectors. The objective in our experiment was for the detectors to identify the concept boundaries. When the concept changes from one class to another, we record whether this change point was detected. With this scheme, if we are experiencing a long-lived concept such as a denial of service attack then after a sufficient number of examples of the same concept, we would expect the change detection methods to also detect the changepoint back to the normal class, or to another attack.

One challenge for the change detectors in this interpretation is that some concepts may be very short-lived, that is, the change in the distribution is a 'blip', involving only a few observations, after which the distribution reverts back to the original one. Such blips may be too short to allow for detection by any method which is not looking for isolated outliers.

\section{Results and Discussion}

Figure 3 visualises the ARL/TTD space for abrupt and gradual change type by the categories in the taxonomy by Gama et al. [18]. Each plot contains all 96 points (one for each data set) of the 88 change detection methods. Empirically, there is a clear and visible distinction between the methods in the Control Chart category, which performed, on average, worse than chance, and those in the other two categories. Table 5 confirms that Sequential Analysis and Monitoring distribution methods were much more likely to exhibit a high ARL. Furthermore, distribution monitoring methods exhibited considerably lower TTD whilst being competitive on ARL with Sequential Analysis methods. Observe the two distinct clusters in the ARL/TTD space for this category (the triangle marker), and the relative sparsity in-between. We suspect that this is the effect of gradual change on the TTD statistic. This is visible between the figures, where we observe that, in the gradual change experiment, those methods with a high ARL and low TTD struggle to better a TTD of 50, which is the halfway point of introducing the gradual change. Those methods with an already low ARL do not move significantly in the TTD axis between experiments. We suspect that this is because a low ARL implies an over-eager detector, which in turn increases the probability that a valid detection is due to random chance rather than a response to observation of the data.

The bottom two charts in Figure 3 visualise the NFA/MDR space for the aforementioned categories. Interestingly, we see a very similar effect for control chart methods. To understand why the performance of this category is so poor, we must consider the assumptions of the detectors. This experiment presented the data points directly to the change detection methods in the ensemble. Specifically, this category contains EDDM, $\mathrm{HDDM}_{A}$ and $\mathrm{HDDM}_{W}$, all of which share a common ancestor in DDM. Whilst the MOA interface for change detectors accepts 64 bit floating point numbers, these methods were not intended for continuous-valued data. As we mention in subsection 3.1.2, DDM assumes the Binomial distribution. It also assumes that the monitored value (e.g., error rate of a classifier) will decrease while the underlying distribution is stationary. The derived methods also share this assumption, which is fundamentally violated by the nature of the data presented to them in this experiment.

The top 20 performers averaged over abrupt and gradual change are summarised in the left half of Table 6 . The performers were 

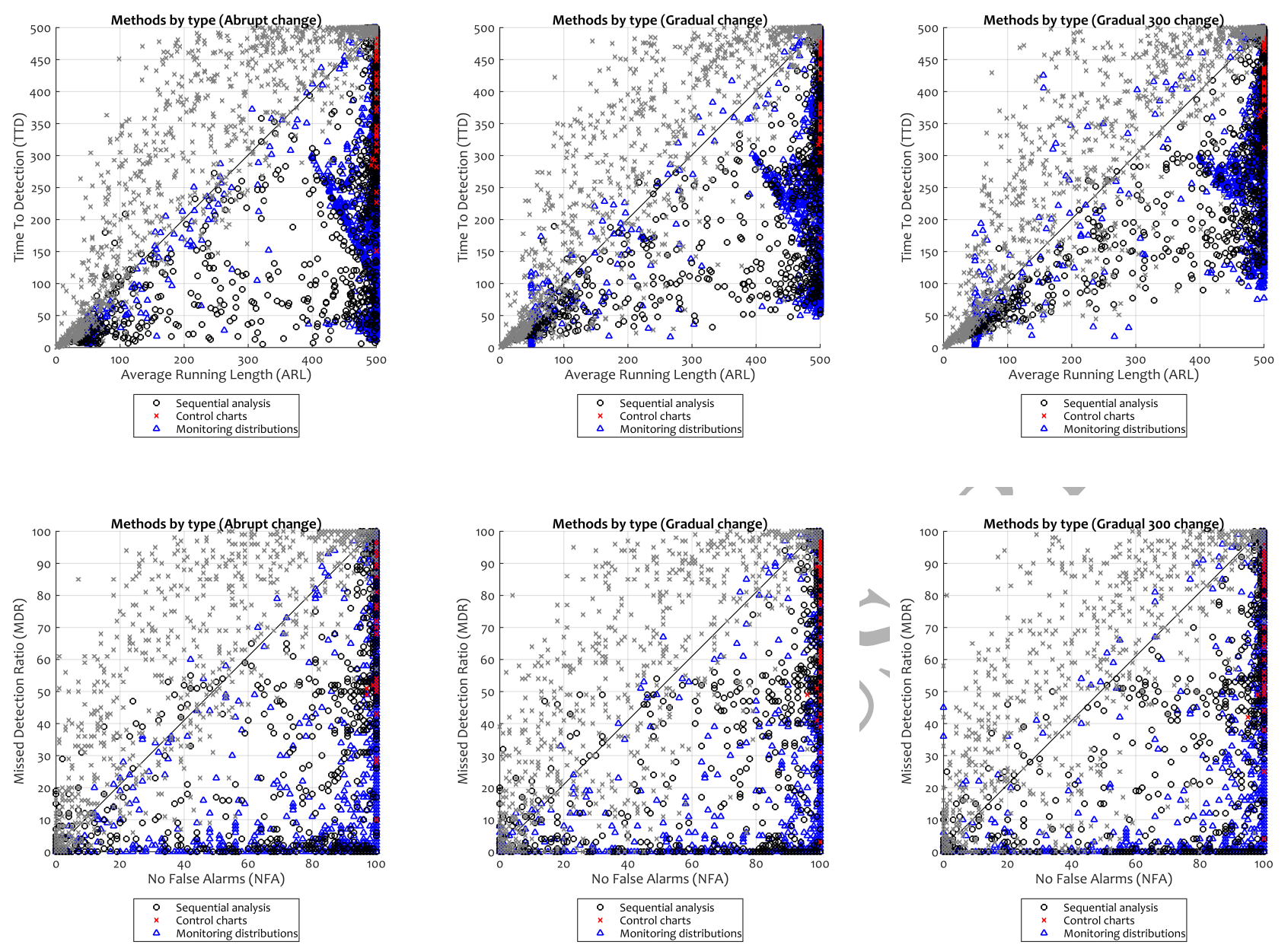

Figure 3: The three categories of detector, visualised in the ARL/TTD space for the abrupt, gradual 100 and gradual 300 change experiments, respectively. Data points for methods whose assumptions were violated are greyed out, but retain their category marker.

\begin{tabular}{|c|c|c|c|c|c|c|c|c|}
\hline \multirow{2}{*}{ Method } & \multicolumn{2}{|c|}{ ARL } & \multicolumn{2}{|c|}{ TTD } & \multicolumn{2}{|c|}{ NFA } & \multicolumn{2}{|c|}{ MDR } \\
\hline & $\mu$ & $\sigma$ & $\mu$ & $\sigma$ & $\mu$ & $\sigma$ & $\mu$ & $\sigma$ \\
\hline Sequential analysis & 433.49 & 134.28 & 323.02 & 187.07 & 80.21 & 34.26 & 59.14 & 42.26 \\
\hline Control charts & 499.93 & 0.68 & 486.67 & 46.14 & 99.97 & 0.28 & 96.46 & 12.02 \\
\hline Monitoring distributions & 435.16 & 145.36 & 219.77 & 176.18 & 81.07 & 34.73 & 29.75 & 38.82 \\
\hline
\end{tabular}

ranked by minimum euclidean distance to the ideal points in the ARL/TTD and NFA/MDR spaces, $(500,0)$ and $(1,0)$.

The results for each individual method are summarised in the ARL/TTD space in Figure 4, and in the NFA/MDR space in Figure 5. In the ARL/TTD space, the SEED and ADWIN detectors were the best performers, with Page Hinkley, CUSUM2 and SEQ1 showing competitive patterns. The multivariate detectors exhibited a large standard deviation, suggesting that their performance is related to the suitability of the data - an observation which would appear to lend further credence to the conclusions of Allipi et al. [17], as well as our own hypothesis. In the NFA/MDR space, the winners are the low quorum ensembles of the SEED and ADWIN detectors. In fact, all the ensembles outside of the control chart category performed favorably compared to the multivariate detectors. Observing the curves of the SEED, ADWIN, Page Hinkley, CUSUM1, CUSUM2 and SEQ1 detectors across both sets of metrics, we see that the ideal agreement threshold is a case-by-case problem. The ADWIN ensemble improves almost linearly as we reduce the agreement threshold, suggesting that the optimum scheme is one whereby any member of the ensemble has absolute authority to signal a change. With other ensembles such as SEED and SEQ1, the $1 \%$ threshold is beyond the optimal, with the best ensembles having thresholds of $5 \%$ and $10 \%$, respectively in the NFA/MDR space. It appears that the optimal choice of threshold differs slightly between the ARL/TTD space and the NFA/MDR space. There is a clear and expected effect between abrupt and gradual change on the ARL/TTD space mostly in the 

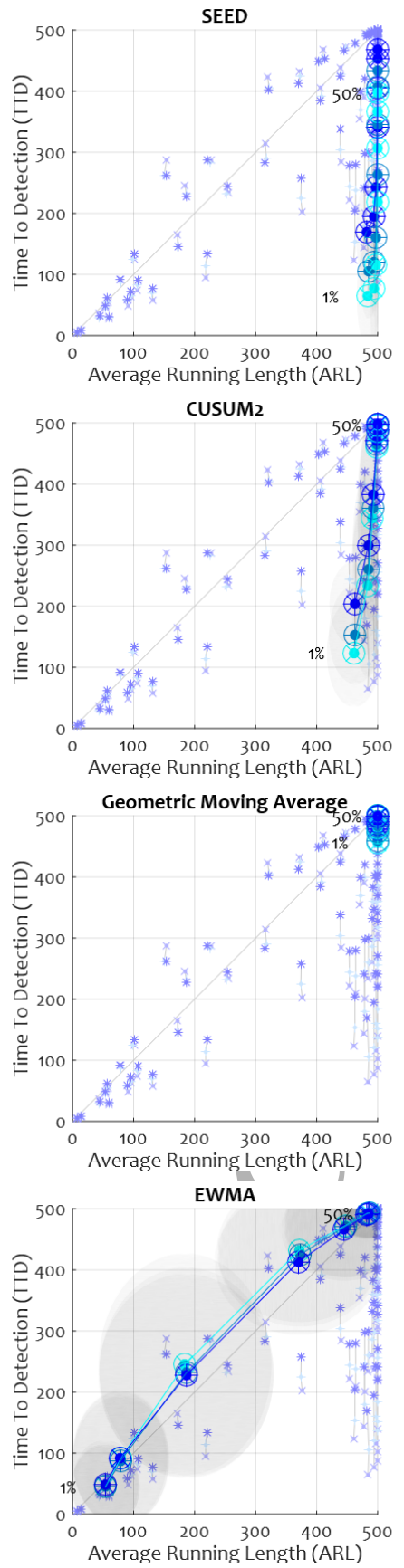
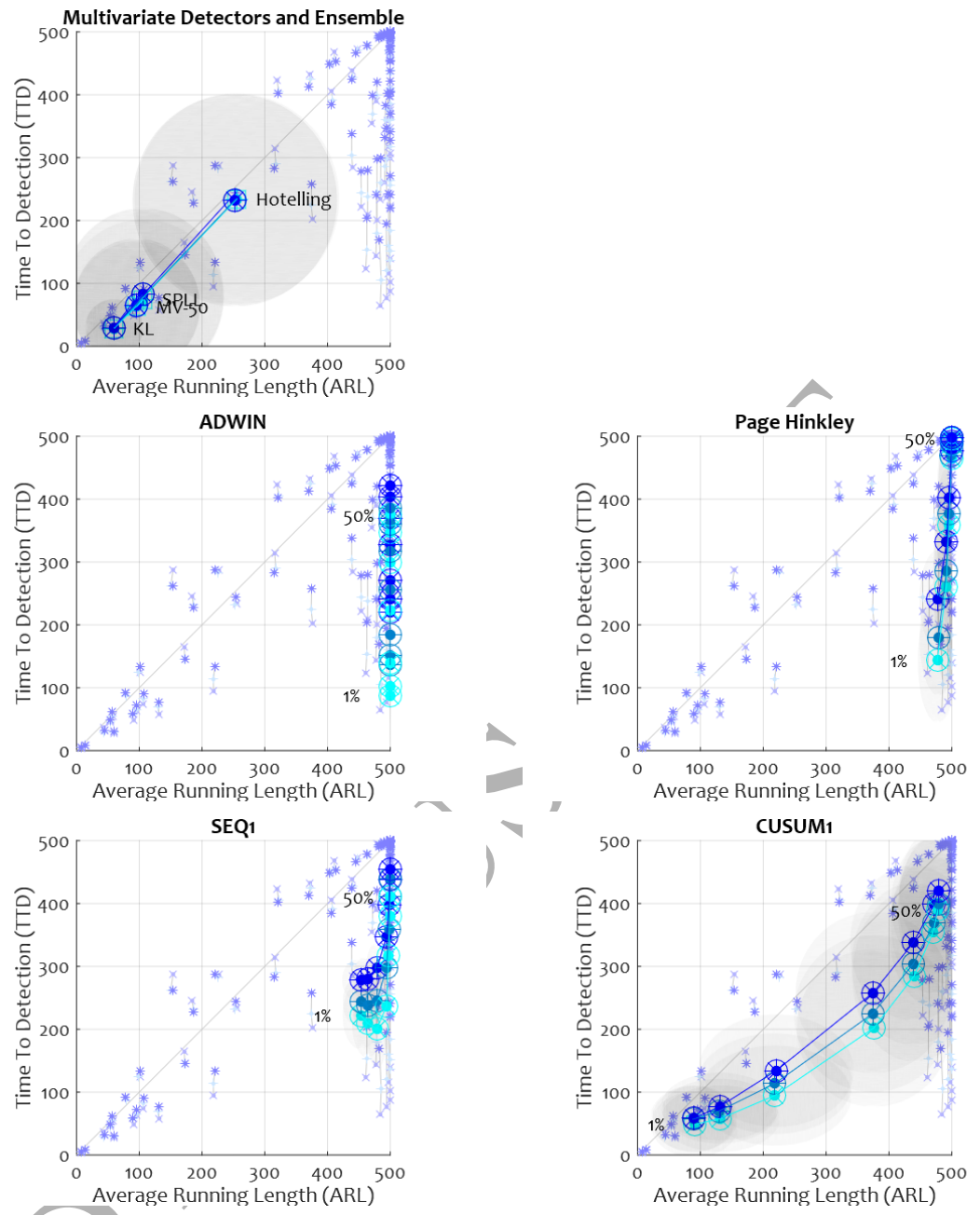

CUSUM
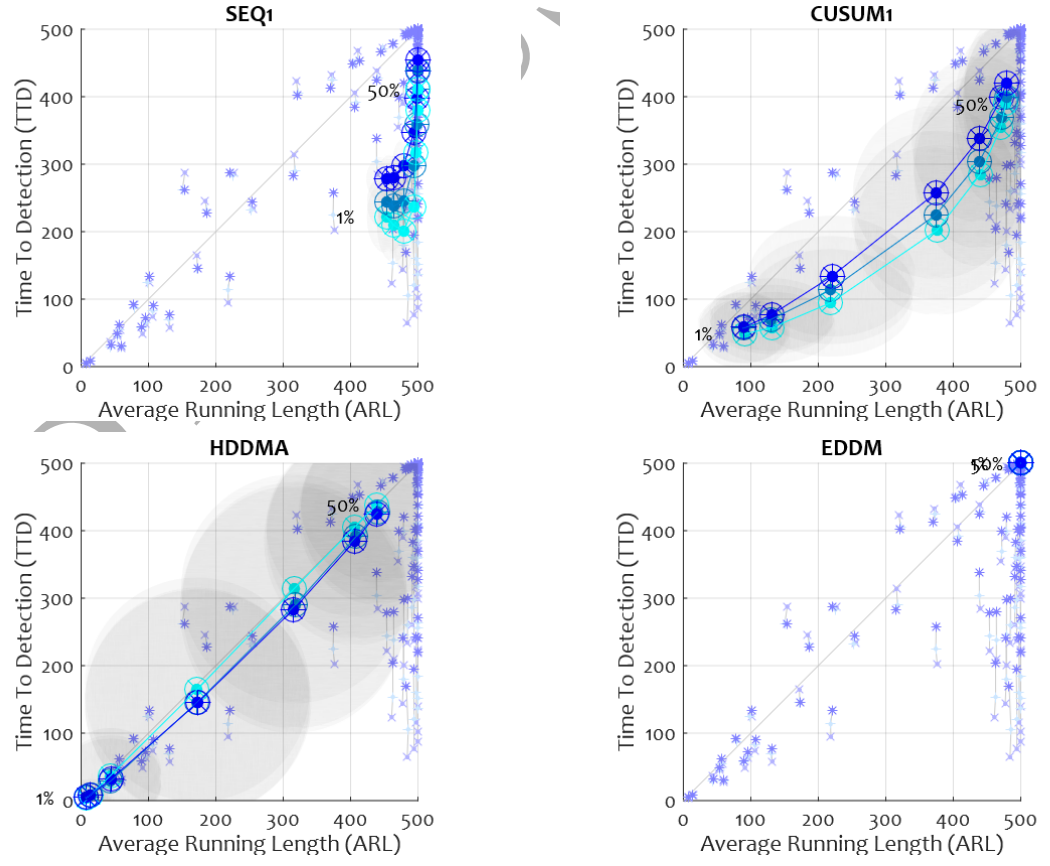

EDDM
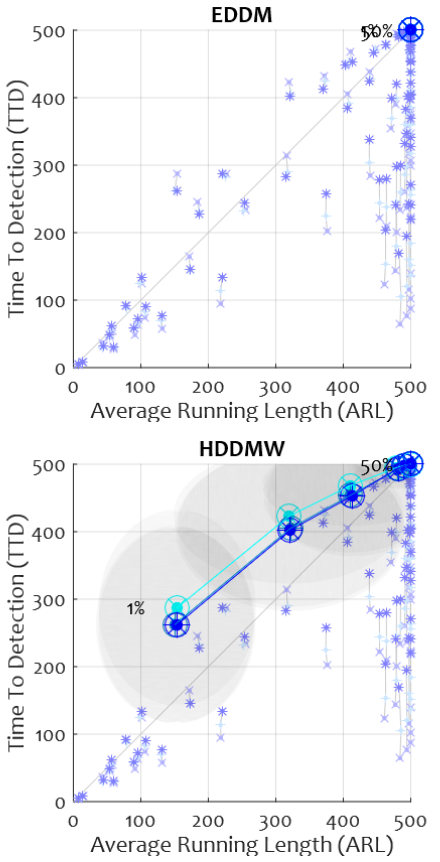

Figure 4: Change detection methods in the space spanned by ARL and TTD for the main experiment. Each method has been examined with different agreement thresholds. Each plot contains 88 gradual and 88 abrupt detector points, averaged across the 96 data sets - gradual 300 as a blue $\mathrm{x}$ (darkest), linked to the paired gradual 100 result as a purple + and the abrupt result as a cyan * (lightest). Each detector's points are highlighted, again in blue, purple and cyan for gradual 300 , gradual and abrupt change type, respectively. The shaded ellipses around the mean detector results are the standard deviations across the 96 datasets. The ideal point is (500,0). 

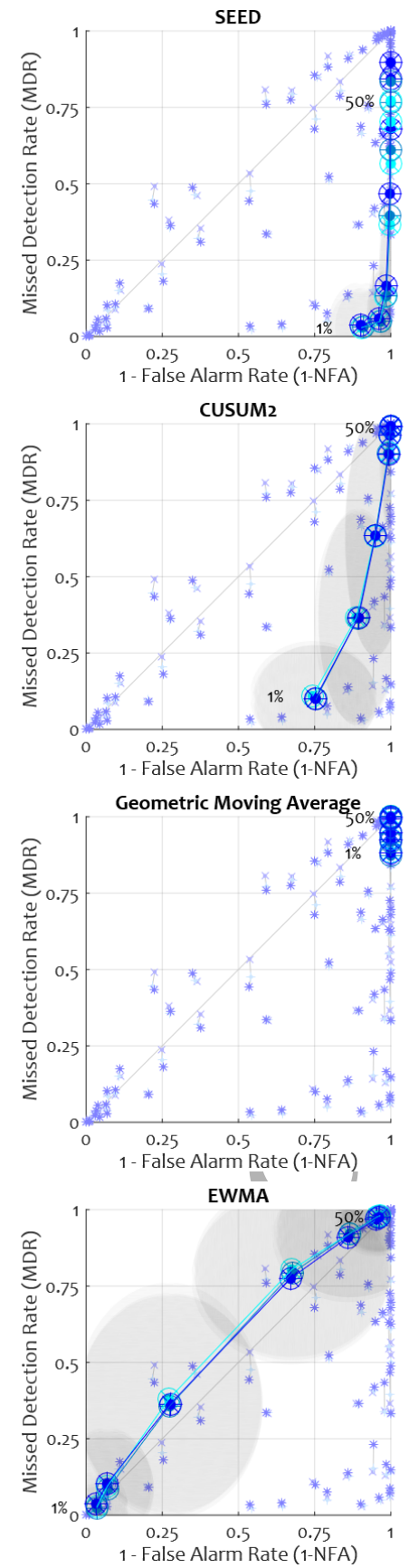
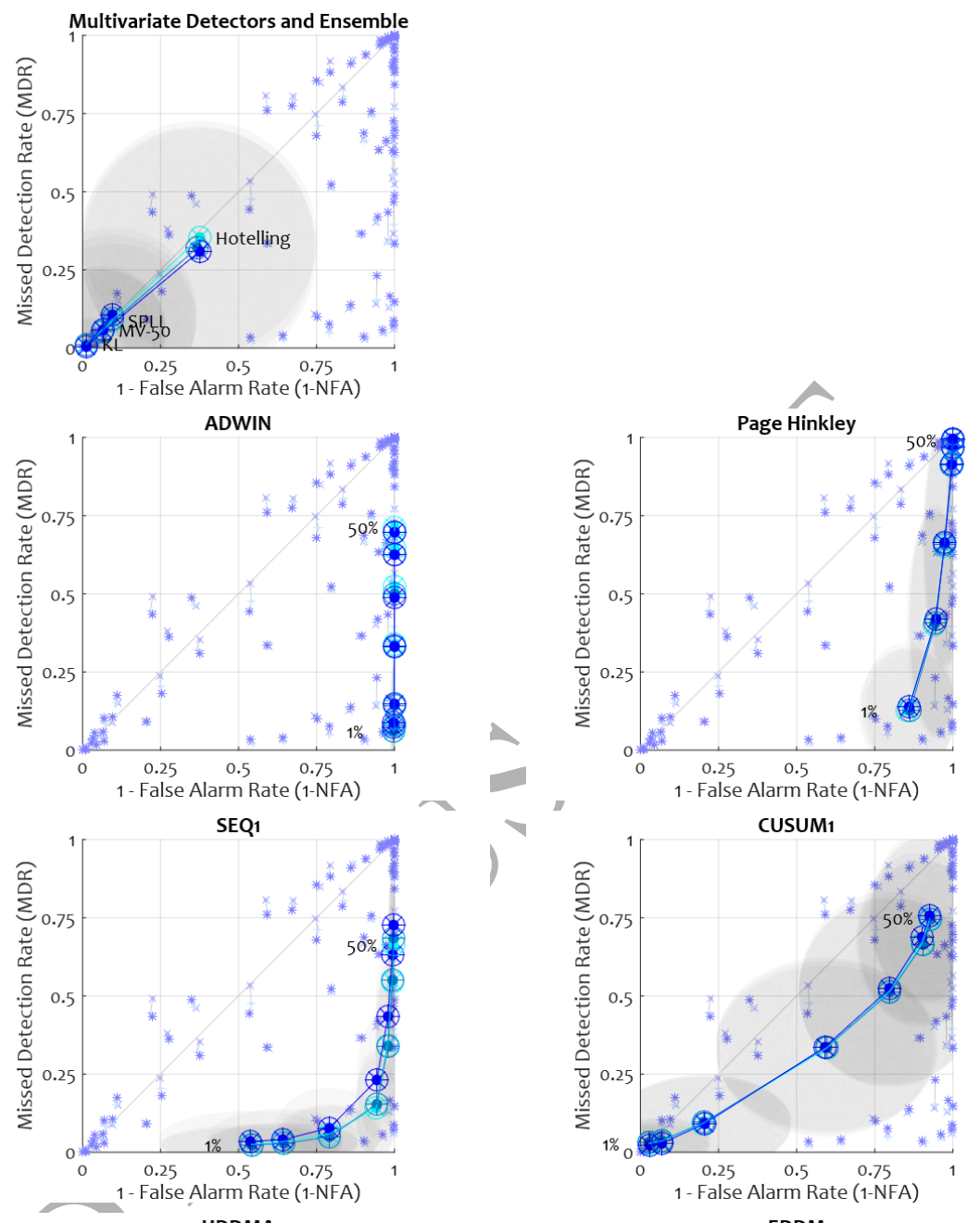

CUSUM1
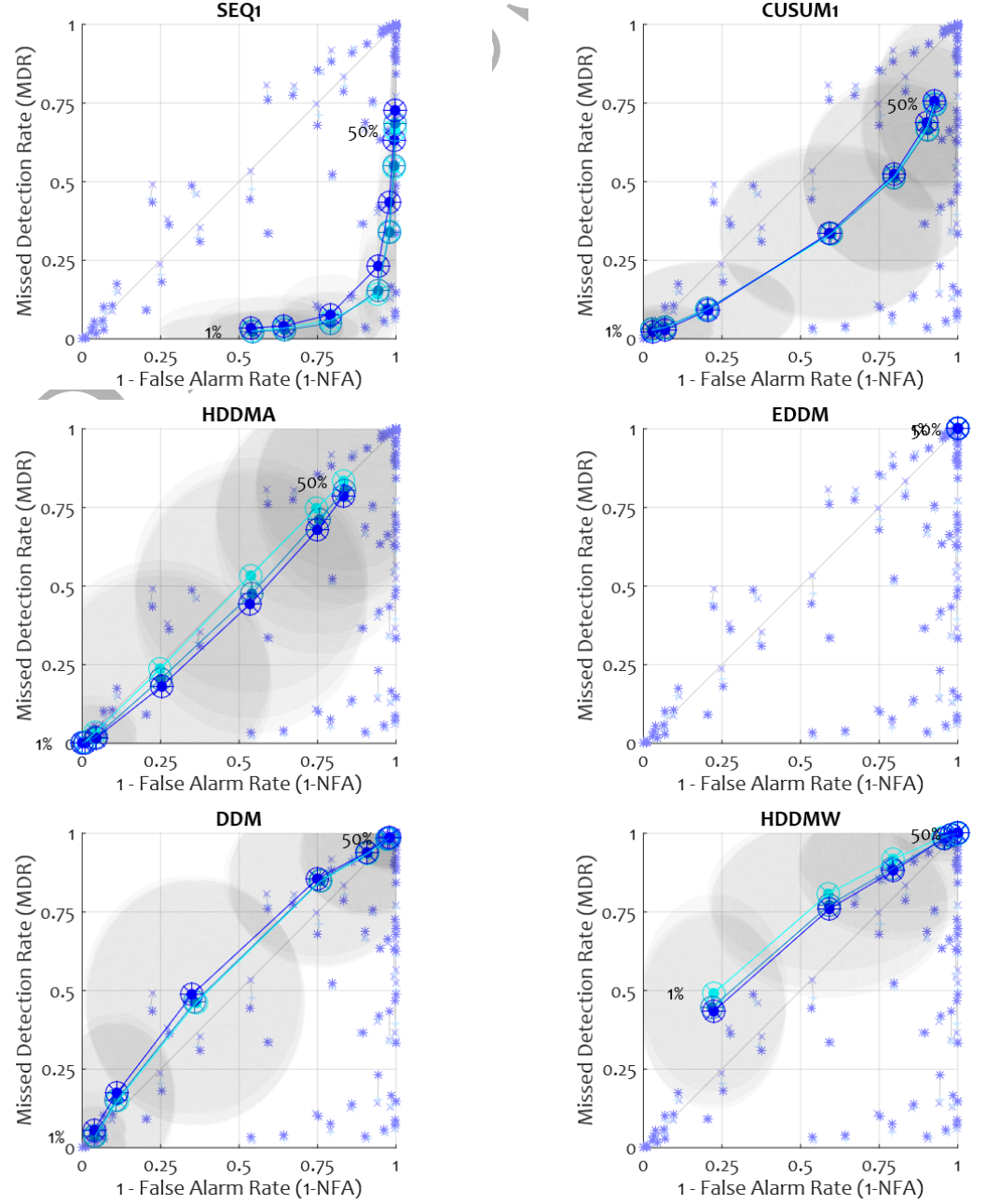

Figure 5: Change detection methods in the space spanned by NFA and MDR for the main experiment. Each method has been examined with different agreement thresholds. Each plot contains 88 gradual and 88 abrupt detector points, averaged across the 96 data sets - gradual 300 as a blue x (darkest), linked to the paired gradual 100 result as a purple + and the abrupt result as a cyan * (lightest). Each detector's points are highlighted, again in blue, purple and cyan for gradual 300 , gradual 100 and abrupt change type, respectively. The shaded ellipses around the mean detector results are the standard deviations across the 96 datasets. The ideal point is $(1,0)$. 
Table 6: The top 20 performers in the main experiment and the case study. The methods are ranked in the listed 2D spaces by minimum euclidean distance to their respective ideal points, $(500,0),(1,0),(7684.09,0)$ and $(0,0)$. The ranks of the multivariate detectors and multivariate ensemble are also shown if they were not represented in the top 20 .

\begin{tabular}{|c|c|c|c|c|c|c|c|c|c|c|c|c|}
\hline & \multicolumn{6}{|c|}{ Main Experiment Averages } & \multicolumn{6}{|c|}{ Case Study - KDD Cup 1999} \\
\hline \# & Detector & ARL & TTD & Detector & NFA & MDR & Detector & ARL & TTD & Detector & FPR & MDR \\
\hline 1 & SEED-1 & 484.18 & 113.07 & SEED-5 & 0.96 & 0.05 & ADWIN-20 & 10578.05 & 327.71 & HDDMA-1 & 0.14 & 0.07 \\
\hline 2 & SEED-5 & 494.00 & 130.66 & ADWIN-1 & 1.00 & 0.06 & SEED-20 & 10900.19 & 648.86 & CUSUM1-1 & 0.03 & 0.26 \\
\hline 3 & ADWIN-1 & 499.67 & 148.46 & ADWIN-5 & 1.00 & 0.08 & SEQ1-5 & 10930.04 & 578.64 & CUSUM1-5 & 0.01 & 0.31 \\
\hline 4 & CUSUM2-1 & 462.10 & 160.48 & SEED-1 & 0.91 & 0.03 & CUSUM1-30 & 11153.81 & 1179.54 & HDDMA-5 & 0.03 & 0.31 \\
\hline 5 & ADWIN-5 & 499.91 & 165.00 & SEED-10 & 0.98 & 0.14 & SEQ1-1 & 4291.67 & 180.79 & PH-1 & 0.01 & 0.32 \\
\hline 6 & SEED-10 & 497.54 & 172.90 & ADWIN-10 & 1.00 & 0.15 & CUSUM2-5 & 3462.90 & 1281.90 & CUSUM2-1 & 0.01 & 0.32 \\
\hline 7 & PH-1 & 477.96 & 187.96 & SEQ1-20 & 0.94 & 0.18 & ADWIN-10 & 3094.09 & 85.84 & HDDMW-1 & 0.32 & 0.08 \\
\hline 8 & ADWIN-10 & 499.94 & 197.93 & PH-1 & 0.86 & 0.13 & SEED-10 & 2828.08 & 74.83 & GEOMA-1 & 0.01 & 0.33 \\
\hline 9 & SEQ1-5 & 463.90 & 242.38 & SEQ1-10 & 0.79 & 0.06 & DDM-5 & 13724.94 & 1974.68 & MV-50 & 0.02 & 0.36 \\
\hline 10 & SEQ1-10 & 478.91 & 247.84 & CUSUM2-1 & 0.75 & 0.10 & HDDMA-10 & 2605.21 & 3357.99 & Hotelling & 0.02 & 0.36 \\
\hline 11 & SEQ1-1 & 453.59 & 248.14 & ADWIN-20 & 1.00 & 0.33 & CUSUM1-20 & 2646.27 & 3734.60 & EDDM-1 & 0.00 & 0.37 \\
\hline 12 & CUSUM1-20 & 374.97 & 228.50 & SEQ1-5 & 0.64 & 0.03 & ADWIN-5 & 741.15 & 48.96 & ISUM1-10 & 0.00 & 0.37 \\
\hline 13 & CUSUM2-5 & 484.61 & 264.66 & SEQ1-30 & 0.98 & 0.37 & SEED-5 & 682.78 & 48.51 & $\mathrm{KL}$ & 0.01 & 0.37 \\
\hline 14 & ADWIN-20 & 499.99 & 268.90 & CUSUM2-5 & 0.89 & 0.37 & EDDM-1 & 563.22 & 39.67 & SPLL & 0.02 & 0.37 \\
\hline 15 & SEED-20 & 499.52 & 274.41 & SEED-20 & 1.00 & 0.41 & DDM-1 & 441.54 & 1494.63 & EWMA-1 & 0.00 & 0.39 \\
\hline 16 & PH-5 & 491.43 & 293.04 & PH-5 & 0.94 & 0.41 & EWMA-1 & 541.86 & 2015.76 & DDM-1 & 0.00 & 0.39 \\
\hline 17 & SEQ1-20 & 494.19 & 294.23 & SEQ1-1 & 0.54 & 0.03 & SEED-1 & 229.07 & 32.73 & ADWIN-1 & 0.01 & 0.40 \\
\hline 18 & CUSUM1-10 & 219.09 & 114.13 & ADWIN-30 & 1.00 & 0.50 & ADWIN-1 & 187.46 & 24.95 & SEED-1 & 0.00 & 0.41 \\
\hline 19 & CUSUM1-30 & 439.02 & 308.69 & CUSUM1-20 & 0.59 & 0.34 & PH-1 & 113.59 & 15.71 & SEED-5 & 0.00 & 0.43 \\
\hline 20 & ADWIN-30 & 499.99 & 328.74 & CUSUM1-30 & 0.80 & 0.52 & GEOMA-1 & 108.70 & 19.54 & ADWIN-5 & 0.00 & 0.44 \\
\hline
\end{tabular}

\begin{tabular}{|c|c|cc|c|c|ccc|c|c|c|}
\hline$\#$ & Detector & ARL & TTD & $\#$ & Detector & NFA & MDR & $\#$ & Detector & ARL & TTD \\
\hline 21 & Hotelling & 499.95 & 432.97 & 30 & Hotelling & 0.01 & 0.01 & 23 & KL & $\infty$ & $\infty$ \\
34 & SPLL & 484.61 & 264.66 & 47 & SPLL & 0.04 & 0.04 & 25 & SPLL & $\infty$ & $\infty$ \\
39 & MV-50 & 499.88 & 497.29 & 54 & MV-50 & 1.00 & 1.00 & 26 & MY-50 & 541.86 & 2015.76 \\
47 & KL & 57.02 & 56.73 & 68 & KL & 0.86 & 0.13 & 27 & Hotelling & 9137.79 & 8020.57 \\
\hline
\end{tabular}

TTD axis, with TTD being marginally lower for abrupt changes in those detectors whose assumptions are not violated.

Bearing in mind the works of Allipi et al. [17] and Evangelista et al. [16], we were interested in observing the effects of data dimensionality on the missed detection rate. Scatterplots of average missed detection rate against dataset dimensionality, for each category of ensemble and for the multivariate detectors, are presented in Figure 6. The scatter patterns suggest that changes in higher-dimensional spaces are more likely to be missed.

\subsection{The Case Study}

The right half of Table 6 summarises the top 20 performers on the case study data. As this experiment was a single run, we present the false positive rate as FPR, instead of the NFA measure. The methods were ranked by the minimum euclidean distance to the ideal points $(7864.09,0)$ and $(0,0)$ for the ARL/TTD and FPR/MDR spaces respectively. The ideal ARL of 7864.09 was calculated by observing the ARL of a perfect, 'cheating' detector, which signalled immediately for all changepoints and recorded no false positives. We see a familiar pattern in the ARL/TTD space, with the SEED, ADWIN and CUSUM-based methods well represented within the top 20. In the FPR/MDR space, the winners are primarily low-threshold ensembles. We note that 8 methods; ADWIN-1, ADWIN-5, SEED-1, SEED-5, EDDM-1, PH-1, GEOMA-1 and EWMA-1 are represented in the top 20 in both spaces. We also observe that the top ranked ensembles across the two spaces here differed modestly from the top performers in the main experiment with the simulated abrupt and gradual changes. The improvement in performance of control chart-based methods may be due to the incidence of a number of contextually important binary features in this dataset. The best performing multivariate detectors were ranked 23rd and 9th in the two spaces respectively. Apart from the high false positive rates of $\mathrm{HDDM}_{W}-1$ and $\mathrm{HDDM}_{A}-1$, the ensembles were competitive or better than the multivariate detectors on TTD and MDR, and generally exhibited less false positives. The dominance of the low-threshold ensembles mirrors their success in the previous experiment, and suggests that between $1 \%$ and $5 \%$ agreement is a sensible starting point when employing this scheme, across a range of different detectors.

\section{Conclusions}

The results of the experiment and the case study demonstrate the viability of ensemble combination of univariate change detectors over multivariate data. Over 96 datasets, ensemble methods frequently outperformed multivariate detectors in all metrics, especially at low agreement thresholds. The multivariate detectors did not even feature in the top 20 overall performers in either space, as seen in Table 6 . This would appear to tally with the conclusions of Evangelista et al. [16]. The SEED and ADWIN detectors appear to be the best suited to ensemble combination in this manner. Given that the SEQ1 algorithm is an ADWIN-derivative, we would expect it to exhibit a similar performance. We see that it does exhibit very similar performance to the ADWIN ensembles in terms of missed detections, but it signals far more eagerly for a higher rate of false 

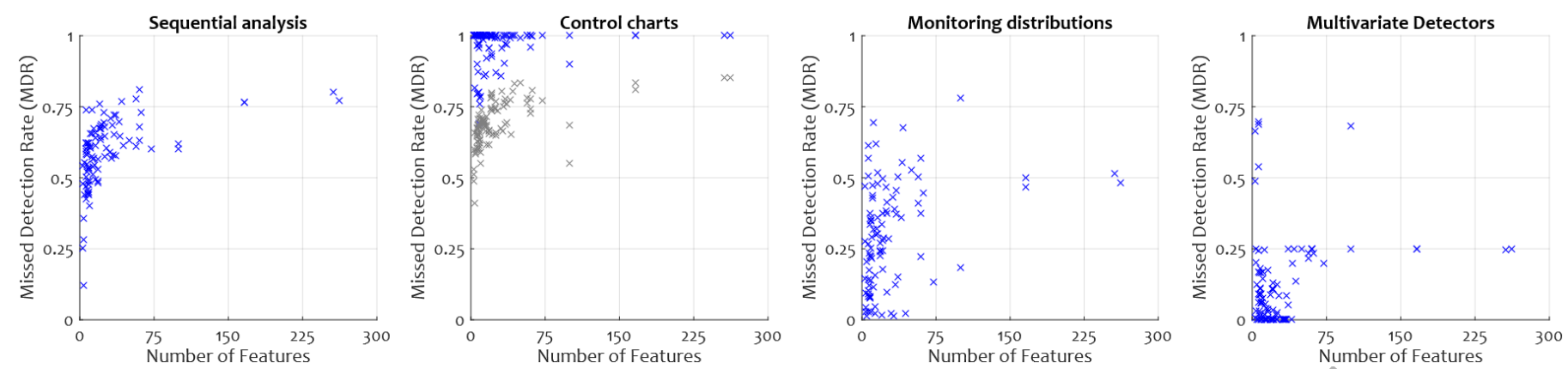

Figure 6: Scatter plots of dataset dimensionality against average missed detection rate for the 96 datasets. The plots are arranged by the category of the detectors. Data points from detectors with violated assumptions are greyed out.

alarms. This may be a reflection, as we noted in section 3.1.3, of the authors' choice of the Bernstein bound over the more conservative Hoeffding bound to set the threshold.

Those detectors which make strong assumptions on the basis that they are monitoring the error stream of an attached learner were unsurprisingly poor when applied to raw data in this scheme. This accounts for the worse-than-chance performances of the $\mathrm{HDDM}_{A}$, $\mathrm{HDDM}_{W}$, EDDM, DDM and EWMA methods.

Upon observation of the results, we note that the ideal agreement threshold varies between detectors. The curves in Figures 4 and 5 can be used to pick a suitable threshold for each of the successful detectors. Taking ADWIN for example, the lack of movement on the false alarm rate relative to the threshold changes suggests that an ensemble might be close to optimal if any member is given absolute authority for signalling. As a counter example, the SEQ1 ensembles seem to have an optimal agreement threshold of between $10 \%$ and $20 \%$.

We observed empirically that all categories of detectors exhibited a positive relationship between missed detections and dataset dimensionality, as Allipi et al. [17] suggest, albeit to varying degrees. Evangelista et al. [16] also state that unsupervised learning in subspaces of the data is a means to address the curse of dimensionality. This is not strongly reflected in Figure 6, with the multivariate detectors appearing to exhibit the weakest relationship of missed detections with dimensionality. However, the ensembles had a much wider spread of results, and the better ensembles considerably outperformed the multivariate detectors.

The experimental results invite many avenues of future work. The application of existing work on feature extraction, weighting or selection could change the optimal ensemble thresholds by removing redundant features. Ensembles could be tailored to the type and rate of the expected changes in the data stream, incorporating domain-specific knowledge rather than the generic approach here. The numerous univariate change detection approaches not considered within this paper can be evaluated in similarly constructed ensembles.

\section{Acknowledgment}

This work was done under project RPG-2015-188 funded by The Leverhulme Trust, UK; Spanish Ministry of Economy and Competitiveness through project TIN 2015-67534-P and the
Spanish Ministry of Education, Culture and Sport through Mobility Grant PRX16/00495. The 96 datasets were originally curated for use in the work of Fernández-Delgado et al. [53] and accessed from the personal web page of the author ${ }^{5}$. The KDD Cup 1999 dataset used in the case study was accessed from the UCI Machine Learning Repository [10].

\section{References}

[1] E. Page, "Continuous inspection schemes," Biometrika, vol. 41, no. 1, pp. 100-115, 1954.

[2] A. Wald, Sequential Analysis. New York: Dover Publications, 1974.

[3] M. Basseville and I. V. Nikiforov, Detection of abrupt changes: theory and application. Englewood Cliffs: Prentice Hall, 1993, vol. 104.

[4] M. Markou and S. Singh, "Novelty detection: A review - Part 1: Statistical approaches," Signal Processing, vol. 83, no. 12, pp. 2481-2497, 2003.

[5] M. A. F. Pimentel, D. A. Clifton, L. Clifton, and L. Tarassenko, "A review of novelty detection,” Signal Processing, vol. 99, pp. 215-249, 2014.

[6] I. Ben-gal, "Outlier Detection," Data Mining and Knowledge Discovery Handbook, pp. 131-146, 2005. [Online]. Available: http://www.eng.tau.ac.il/ bengal/outlier.pdf

[7] A. Bifet, G. Holmes, R. Kirkby, and B. Pfahringer, "MOA Massive Online Analysis," Journal of Machine Learning Research, vol. 11, pp. 1601-1604, 2011. [Online]. Available: http://moa.cs.waikato.ac.nz/details/

[8] A. Bifet, J. Read, B. Pfahringer, G. Holmes, and I. Žliobaite, "CD-MOA: Change detection framework for massive online analysis," Advances in Intelligent Data Analysis XII, pp. 92-103, 2013.

[9] L. Kuncheva, "Classifier ensembles for detecting concept change in streaming data: Overview and perspectives," in Proceedings of the Second Workshop SUEMA, ECAI 2008, 2008, pp. 5-9. [Online]. Available: http://hdl.handle.net/10242/41809

[10] M. Lichman, “ $\{U C I\}$ Machine Learning Repository,” 2013. [Online]. Available: http://archive.ics.uci.edu/ml

[11] L. I. Kuncheva, "Change detection in streaming Multivariate data using likelihood detectors," IEEE Transactions on Knowledge and Data Engineering, vol. 25, no. 5, pp. 1175-1180, 2013.

[12] F. Zorriassatine, A. Al-Habaibeh, R. M. Parkin, M. R. Jackson, and J. Coy, "Novelty detection for practical pattern recognition in condition monitoring of multivariate processes: A case study," International Journal of Advanced Manufacturing Technology, vol. 25, no. 9-10, pp. 954-963, 2005.

[13] D. Agarwal, "An Empirical Bayes approach to detect anomalies in dynamic multidimensional arrays," in Proceedings - IEEE International Conference on Data Mining, ICDM, 2005, pp. 26-33.

[14] T. D. Nguyen, M. C. Du Plessis, T. Kanamori, and M. Sugiyama, "Constrained least-squares density-difference estimation," IEICE TRANSACTIONS on Information and Systems, vol. 97, pp. 1822-1829, 2014.

\footnotetext{
${ }^{5}$ http://persoal.citius.usc.es/manuel.fernandez.delgado/ papers/jmlr/
} 
[15] A. G. Tartakovsky, B. L. Rozovskii, R. B. Blažek, and H. Kim, "Detection of intrusions in information systems by sequential change-point methods," Statistical Methodology, vol. 3, no. 3, pp. 252-293, 2006.

[16] P. F. Evangelista, M. J. Embrechts, and B. K. Szymanski, "Taming the curse of dimensionality in kernels and novelty detection," in Applied soft computing technologies: The challenge of complexity. Springer, 2006, pp. 425-438.

[17] C. Alippi, G. Boracchi, D. Carrera, and M. Roveri, "Change detection in multivariate datastreams: likelihood and detectability loss," in Proceedings of the Twenty-Fifth International Joint Conference on Artificial Intelligence. AAAI Press, 2016, pp. 1368-1374.

[18] J. Gama, I. Žliobaite, A. Bifet, M. Pechenizkiy, and A. Bouchachia, “A survey on concept drift adaptation," ACM Computing Surveys, vol. 46, no. 4, pp. 1-37, 2014

[19] G. Ditzler, M. Roveri, C. Alippi, and R. Polikar, "Learning in nonstationary environments: A survey," IEEE Computational Intelligence Magazine, vol. 10 no. 4, pp. 12-25, 2015

[20] L. Tarassenko, A. Hann, and D. Young, "Integrated monitoring and analysis for early warning of patient deterioration." British journal of anaesthesia vol. 97, no. 1, pp. 64-8, jul 2006.

[21] X. Song, M. Wu, C. Jermaine, and S. Ranka, "Statistical change detection for multi-dimensional data," Proceedings of the 13th ACM SIGKDD international conference on Knowledge discovery and data mining - KDD '07, vol. V, p. 667, 2007. [Online]. Available: http://portal.acm.org/citation.cfm?doid=1281192.1281264

[22] T. Dasu, S. Krishnan, S. Venkatasubramanian, and K. Yi, "An informationtheoretic approach to detecting changes in multi-dimensional data streams," in Proc. Symp. on the Interface of Statistics, Computing Science, and Applications, 2006.

[23] G. Krempl, Z. F. Siddiqui, and M. Spiliopoulou, "Online clustering of high-dimensional trajectories under concept drift," in Joint European Conference on Machine Learning and Knowledge Discovery in Databases Springer, 2011, pp. 261-276.

[24] M. M. Gaber and P. S. Yu, "Classification of changes in evolving data streams using online clustering result deviation," in Proc. Of International Workshop on Knowledge Discovery in Data Streams, 2006.

[25] H. Hotelling, "The generalization of Student's ratio," in Breakthroughs in Statistics, 1992 , pp. $54-65$

[26] W. J. Faithfull and L. I. Kuncheva, "On optimum thresholding of multivariate change detectors," in Lecture Notes in Computer Science (including subseries Lecture Notes in Artificial Intelligence and Lecture Notes in Bioinformatics), vol. 8621 LNCS. Springer Verlag, 2014, pp. 364-373.

[27] B. Krawczyk, L. L. Minku, J. Gama, J. Stefanowski, and M. Woźniak, "Ensemble learning for data stream analysis: a survey," Information Fusion, vol. 37, pp. 132-156, 2017.

[28] H. M. Gomes, J. P. Barddal, F. Enembreck, and A. Bifet, "A survey on ensemble learning for data stream classification," ACM Computing Surveys (CSUR), vol. 50, no. 2, p. 23, 2017.

[29] B. I. F. Maciel, S. G. T. C. Santos, and R. S. M. Barros, "A Lightweight Concept Drift Detection Ensemble," in 2015 IEEE 27th International Conference on Tools with Artificial Intelligence (ICTAI). IEEE, nov 2015, pp. 1061-1068. [Online]. Available: http://ieeexplore.iee.org/lpdocs/epic03/wrapper.htm?arnumber=7372248

[30] M. Woźniak, P. Ksieniewicz, B. Cyyganek, and K. Walkowiak, "Ensembles of heterogeneous concept drift detectors-experimental study," in IFIP International Conference on Computer Information Systems and Industrial Management, ser. Lecture Notes in Computer Science, vol. 9842. Springer, 2016, pp. 538-549.

[31] L. Du, Q. Song, L. Zhu, and X. Zhu, "A selective detector ensemble for concept drift detection," The Computer Journal, vol. 58, no. 3, pp. 457-471, 2014.

[32] C. Alippi, G. Boracchi, and M. Roveri, "Hierarchical change-detection tests," IEEE transactions on neural networks and learning systems, vol. 28, no. 2, pp. 246-258, 2017.

[33] A. Bifet, G. Holmes, B. Pfahringer, R. Kirkby, and R. Gavaldà, "New ensemble methods for evolving data streams," in Proceedings of the 15th ACM SIGKDD International Conference on Knowledge Discovery and Data Mining. ACM 2009, pp. 139-148.

[34] A. Bifet, E. Frank, G. Holmes, and B. Pfahringer, "Accurate ensembles for data streams: Combining restricted Hoeffding trees using stacking." 2nd Asian Conference on Machine Learning (ACML2010), pp. 1-16, 2010. [Online]. Available: http://eprints.pascal-network.org/archive/00007198/

[35] _ - "Ensembles of restricted hoeffding trees," Proceedings of the 14th
International Conference on Artificial Intelligence and Statistics, vol. 15, no. 212, pp. 434-442, 2012.

[36] I. Frias-Blanco, J. del Campo-Avila, G. Ramos-Jimenez, R. Morales-Bueno, A. Ortiz-Diaz, and Y. Caballero-Mota, "Online and non-parametric drift detection methods based on Hoeffding's bounds," IEEE Transactions on Knowledge and Data Engineering, vol. 27, no. 3, pp. 810-823, 2015.

[37] J. Gama, P. Medas, G. Castillo, and P. Rodrigues, "Learning with drift detection," Advances in Artificial Intelligence - SBIA 2004, pp. 286-295, 2004

[38] M. Baena-García, J. del Campo Ávila, R. Fidalgo, A. Bifet, R. Gavalda, and R. Morales-Bueno, "Early Drift Detection Method," Fourth international workshop on knowledge discovery from data streams, vol. 6, pp. 77-86, 2006.

[39] W. N. Street and Y. Kim, "A streaming ensemble algorithm (SEA) for large-scale classification," Proceedings of the seventh ACM SIGKDD international conference on Knowledge discovery and data mining - KDD '01, vol. 4, pp. 377-382, 2001. [Online]. Available: http://portal.acm.org/citation.cfm?doid $=502512.502568$

[40] D. T. J. Huang, Y. S. Koh, G. Dobbie, and R. Pears, "Detecting volatility shift in data streams," in Data Mining (ICDM), 2014 IEEE International Conference on. IEEE, 2014, pp. 863-868.

[41] A. Bifet and R. Gavaldà, "Learning from time-changing data with Adaptive Windowing," Proceedings of the 2007 SIAM International Conference on Data Mining, pp. 443-448, 2007.

[42] S. Sakthithasan, R. Pears, and Y. S. Koh, "One pass concept change detection for data streams," Advances in Knowledge Discovery and Data Mining, pp. 461-472, 2013.

[43] G. J. Ross, N. M. Adams, D. K. Tasoulis, and D. J. Hand, "Exponentially weighted moving average charts for detecting concept drift," Pattern Recognition Letters, vol. 33, no. 2, pp. 191-198, 2012

[44] S. W. Roberts, "Control chart tests based on geometric moving averages," Technometrics, vol. 3, pp. 239-250, 2012. [Online]. Available: http://dx.doi.org/10.2307/1271439

[45] H. Mouss, D. Mouss, N. Mouss, and L. Sefouhi, "Test of page-hinckley, an approach for fault detection in an agro-alimentary production system," in Proceedings of the 5th Asian Control Conference, 2004, pp. 815-818.

[46] J. Gama, R. Sebastião, and P. P. Rodrigues, "On evaluating stream learning algorithms," Machine learning, vol. 90, no. 3, pp. 317-346, 2013.

[47] R. Klinkenberg and T. Joachims, "Detecting concept drift with support vector machines," in Proceedings of ICML-00, 17th International Conference on Machine Learning, 2000, pp. 487-494.

[48] G. Widmer and M. Kubat, "Learning in the presence of concept drift and hidden contexts," Machine Learning, vol. 23, no. 1, pp. 69-101, 1996.

[49] L. Kuncheva, "That elusive diversity in classifier ensembles," in Iberian Conference on Pattern Recognition and Image, vol. 2652. Springer, 2003, pp. 1126-1138. [Online]. Available: http://link.springer.com/chapter/10.1007/978-3-540-44871-6_130

[50] L. I. Kuncheva, Combining Pattern Classifiers: Methods and Algorithms: Second Edition. Wiley Blackwell, sep 2014.

[51] D. Brzezinski and J. Stefanowski, "Ensemble diversity in evolving data streams," in International Conference on Discovery Science, ser. Lecture Notes in Computer Science, vol. 9956. Springer, 2016, pp. 229-244.

[52] M. Tavallaee, E. Bagheri, W. Lu, and A. A. Ghorbani, "A detailed analysis of the kdd cup 99 data set," in Computational Intelligence for Security and Defense Applications, 2009. CISDA 2009. IEEE Symposium on. IEEE, 2009, pp. 1-6.

[53] M. Fernández-Delgado, E. Cernadas, S. Barro, and D. Amorim, "Do we need hundreds of classifiers to solve real world classification problems," Journal of Machine Learning Research, vol. 15, no. 1, pp. 3133-3181, 2014. 